\section{MedienPädagogik}

www.medienpaed.com
Zeitschrift für

Theorie und Praxis

der Medienbildung

ISSN 1424-3636

Themenheft Nr. 18: Neue Medien und individuelle Leistungsdarstellung Möglichkeiten und Grenzen von ePortfolios und eAssessments

\title{
ePortfolios zwischen Reflexion und Assessment - Erfahrungen aus der Lehrpersonenbildung
}

\author{
Marc Egloffstein, Jan Baierlein und Clemens Frötschl
}

\begin{abstract}
Dieser Beitrag beschreibt den Einsatz von ePortfolios im Rahmen der akademischen Ausbildung von Handelslehrern/-innen. Im Mittelpunkt steht dabei die Frage, ob und in welcher Weise in ePortfolios reflektiert wird und welche Faktoren diese Reflexion begünstigen könnten. Ausgehend von theoretischen Grundlagen und den speziellen Anforderungen des Anwendungskontexts werden zwei exemplarische Szenarios für den ePortfolio-Einsatz im Studium der Wirtschaftspädagogik vorgestellt. Auf Basis einer zweidimensionalen Operationalisierung werden in einer quantitativen Inhaltsanalyse die ePortfolios von N=140 Teilnehmern/-innen hinsichtlich reflexiver Aussagen untersucht. In der Gesamtschau zeigt sich ein überwiegender Anteil an oberflächlicher Reflexion. Tiefgehende Reflexion scheint dagegen begünstigt zu werden, wenn Reflexionsaufträge in die Leistungsbeurteilung eingehen und durch offene Orientierungsfragen unterstützt werden. Diese Ergebnisse können als erste Anhaltspunkte für die reflexionsförderliche Gestaltung von ePortfolios interpretiert werden.
\end{abstract}

\section{Problemstellung}

Ohne Übertreibung kann Portfolio als einer der wesentlichen Trends in der aktuellen pädagogischen Fachdiskussion bezeichnet werden. Ausgehend von (zu diskutierenden) Prämissen wie Eigenverantwortung, Selbstorganisation, Kompetenzorientierung oder Lebenslangem Lernen hat das Portfolio-Konzept an vielen Stellen im institutionellen Lehr-Lern-Kontext von Schule und Hochschule Eingang gefunden. Portfolios werden als «Reforminstrument zur Weiterentwicklung der Lehr-Lern-Kultur betrachtet» (Häcker, 2005, 8), das die traditionell isolierten Unterrichtsfunktionen Lehren, Lernen und Beurteilen integrieren und den funktionalen Widerspruch der Leistungsbeurteilung (Förderung vs. Selektion) im Sinne eines «Alternative Assessment» ein Stück weit abmildern kann. Ähnlich grosse Hoffnungen werden mit ePortfolios verbunden, die - wenngleich in Deutschland vergleichsweise wenig verbreitet (Melis \& Homik, 2007) - nicht zuletzt als Reaktion auf eine neue, medienaffine Generation von Lernenden propagiert werden (Seufert \& Brahm, 2007). Im Kontext der Leistungsdarstellung und -beurteilung wird allen Portfoliovarianten eine wichtige Rolle zugeschrieben: Portfolios sollen die (Selbst-) Reflexion der Lernenden fördern und dadurch reflexive Lernprozesse anstossen. 
Gerade in diesem Punkt besteht allerdings noch Forschungsbedarf - insbesondere auch hinsichtlich der Frage, wie Reflexion operationalisiert und gemessen werden kann (Butler, 2006). Umso grösser erscheint daher die Notwendigkeit, portfoliobasierte Reflexion empirisch zu erforschen. In diesem Beitrag wird eine entsprechende Untersuchung am Beispiel zweier ePortfolio-Szenarios im Studium der Wirtschaftspädagogik vorgestellt.

\section{ePortfolios - Annäherung an vielschichtiges Konzept}

Nicht wenige Unschärfen in der Diskussion um Portfolios im Bildungskontext sind dadurch entstanden, dass nicht in allen Fällen klar wird, inwieweit die zugrunde liegende pädagogische Idee umgesetzt wird. Häcker (2006) geht von zwei grundsätzlichen Sichtweisen auf das Portfoliokonzept aus. Im weiteren Sinne bezeichnet Portfolio eine umfassende Lehr-Lern-Konzeption (Portfolioansatz), die auf Lernerund Prozessorientierung abzielt und das Werkzeug Portfolio als «Lehr-, Lern- und Entwicklungsinstrument» (Häcker, 2006, 36) betrachtet. Im engeren Sinne steht Portfolio für eine alternative Methode der Leistungsbeurteilung, welche die Diskrepanz zwischen Lernprozess und Leistungsbeurteilung überwinden und dabei auch formative Bewertungsaspekte herausstellen will. Kern des Konzepts ist in jedem Fall die Gestaltung eines individuellen Portfolios durch den/die Lernende/n. Dabei handelt es sich um eine zweckgerichtete Sammlung von (zunächst schriftlichen) Arbeiten (= Artefakten), welche den Leistungsstand, die individuellen Bemühungen und die Fortschritte des/der Lernenden auf einem oder mehreren Gebieten zeigt (Paulson, Paulson \& Meyer, 1991; Jarbonegg, 2004; Häcker, 2006). Portfolios dienen also der (in unterschiedlichem Masse) selbstbestimmten Darstellung eigener Kompetenzen anhand von ausgewählten Leistungsprodukten. Typische Elemente von Portfolios sind Lernzielformulierungen, Lern- und Arbeitsdokumente, Beurteilungskriterien, Reflexionen bzgl. Artefakten und des eigenen Lern- und Arbeitsprozesses sowie Rückmeldungen und Feedback von Lehrenden und Mitlernenden (Brouër, 2007).

Eng verwandt sind Portfolios mit Lerntagebüchern: In beiden Fällen handelt es sich um «Selbstberichts- bzw. Selbstreflektionsinstrumente mit Prozesscharakter» (Gläser-Zikuda, 2007, 95). Während Lerntagebücher aber vor allem auf eine regelmässige zeit- oder ereignisgesteuerte Bearbeitung abzielen, stehen bei Portfolios die begründete Selektion von Artefakten sowie die Reflexion von Lernprozessen im Vordergrund.

Bezüglich der Leistungsbeurteilung mit Portfolios werden oft weitere Anforderungen angeführt. So sollen die Beurteilungskriterien im Idealfall kooperativ festgelegt, in jedem Falle aber transparent kommuniziert werden, beispielsweise auf der Grundlage von Beurteilungsrastern oder «rubrics» (Jonassen et al., 2007). Die Fremdbeurteilung durch Lehrpersonen sollte durch Selbstbeurteilung der Lernenden ergänzt werden. Peer-Assessment kann dabei an Feedback-Aufgaben, Self-Assessment an 
individuelle Reflexionsaufträge oder die begründete Auswahl von Artefakten gekoppelt werden. Auf diese Weise kann die Leistungsbeurteilung einen formativen Charakter erhalten. Wichtig ist in jedem Falle die Einbindung der Portfolioarbeit in das Lehr-Lern-Geschehen, was nicht zuletzt auch dialogische Rückmeldungen durch die Lehrenden bedingt. Insgesamt ist Portfolio-Assessment mit einem vergleichsweise hohen Aufwand für Lehrende und Lernende verbunden.

ePortfolios waren ursprünglich eher als technische denn als konzeptuelle Weiterentwicklungen von papierbasierten Portfolios zu sehen, erweitern deren Einsatzspektrum allerdings deutlich. Konkret handelt es sich bei ePortfolios um Sammlungen von elektronischen Dokumenten und Multimedia-Artefakten, die mit speziellen Content-Management-Systemen oder Social-Software-Werkzeugen (Baumgartner, HimpsI \& Zauchner, 2009) realisiert werden. Die Online-Umsetzung des Portfoliokonzepts bringt einige wesentliche Vorteile mit sich (vgl. Butler, 2006): ePortfolios ermöglichen eine (durch Rechte- und Rollenkonzepte steuerbare) Veröffentlichung der Inhalte, Feedbacks und Kommentierungen lassen sich leichter realisieren. Sie bieten darüber hinaus ein hohes Mass an Flexibilität bzgl. der Publikation von Inhalten (Ergänzung, Austausch; Verwendung verschiedener Medientypen), sind nicht an die Linearität papierbasierter Darstellungen gebunden und ermöglichen eine portfolio- und systemübergreifende Vernetzung. ePortfolios können daher auch als elektronische Leistungs- beziehungsweise Bewerbungsmappe oder - im Kontext des lebenslangen Lernens - zur zusammenhängenden Abbildung von Lernbiografien dienen.

Das Konzept Portfolio ist seit jeher von einer begrifflichen Vielfalt geprägt, die eine trennscharfe Binnendifferenzierung erschwert. So berichtet Häcker (2006) von mindestens 30 verschiedenen Realisierungsformen, identifiziert gleichzeitig aber auch die drei modellhaften Ausprägungen «individual», «working» und «showcase portfolio» (Häcker \& Lissmann 2007, 219).

Baumgartner, Himpsl und Zauchner (2009) schliessen in einer umfangreichen ePortfolio-Taxonomie auch solche Anwendungsfälle mit ein, die über den Einsatz in institutionellen Lehr-Lern-Arrangements hinausgehen. So wird hier zwischen den Grundtypen Reflexionsportfolio (auch: Bildungsportfolio, Fokus: innere Entwicklung), Entwicklungsportfolio (Fokus: äussere Entwicklung) und Präsentationsportfolio (Fokus: Aussendarstellung) unterschieden.

\section{ePortfolios und Reflexion}

Die Arbeit mit Portfolios ist eng mit dem Konzept des reflexiven Lernens verbunden (Doig et al., 2006; Hilzensauer, 2008). Nicht umsonst wird der Begriff der Reflexion sowohl mit strukturellen Differenzierungen von Portfolios als auch mit gängigen Ablaufmodellen der Portfolioarbeit (Barrett, 2003; Hornung-Prähauser et al., 2007) verknüpft. Ohne vorhergehende Konzeptualisierung bleibt aber oft im Unklaren, was genau unter Reflexion zu verstehen ist. 
Aus psychologischer Sicht bezeichnet Selbstreflexion die Tätigkeit, das eigene «Handeln und Denken zum Objekt desselben» zu machen (Dörner, 1994, 199). Im Gegensatz zum alltäglichen (Nach-)Denken bezieht sich Reflexion dabei vor allem auf die Auseinandersetzung mit komplexen Problemen (Moon, 2004). Korthagen $(2002,63)$ macht den Bezug zum Lernen deutlich und definiert Reflexion als den mentalen Prozess, «zu versuchen, eine Erfahrung, ein Problem oder bestehendes Wissen oder Einsichten zu strukturieren oder restrukturieren». In Bezug auf Lernprozesse wird der Reflexion dabei sowohl eine «katalysatorische» (beschleunigende) als auch eine «transformatorische» (qualitätssteigernde) Wirkung zugeschrieben (Dilger, 2007, 2f.)

Grundlegend für die Konzeptualisierung von reflexivem Lernen sind die Arbeiten von Dewey, Kolb und Schön. Dewey (1910) betont den Handlungsbezug sowie den sequentiellen Charakter des reflexiven Lernens: ausgehend von unbekannten Situationen werden neue Einsichten durch Bezugnahme auf bestehendes Wissen und vergangene Erfahrungen gewonnen. Eine Umsetzung dieser Ideen in ein konkretes Lehr-Lern-Modell stellt der "Learning Cycle» des «Experiential Learning» dar, in dem konkrete Erfahrungen beobachtet und reflektiert, abstrahiert, in neuen Situationen getestet und schliesslich wieder in konkrete Erfahrungen überführt werden (Kolb, 1984). Schön (1983) differenziert den Handlungsbezug in zeitlicher Hinsicht und unterscheidet zwischen direkter «Reflection in action» und nachgelagerter "Reflection on action», wobei vor allem letztere im Kontext von ePortfolios von Belang ist. Der weite Zeitrahmen (sowohl prospektiv als auch retrospektiv) sowie der der explizite Einbezug von Emotionen (Boud, Keogh \& Walker, 1985) unterscheiden Reflexion von der Metakognition im engeren Sinne. Im pädagogischen Kontext kann sich Reflexion auf unterschiedliche Ebenen beziehen. Mögliche Differenzierungen unterscheiden Ziele, Strategien und Inhalte (Jenert, 2008), Lerngegenstand, Lernhandlung und Lernvermögen (Hilzensauer, 2008) oder Lernverhalten, Lehr-Lern-Arrangement und institutionellen Kontext (Häcker, 2005). In der Praxis sind entsprechende Zuordnungen - anders als eine Operationalisierung nach dem Gegenstand oder Inhalt der Reflexion - aber nur schwer zu treffen. Einige Konzeptualisierungen von Reflexion unterscheiden zudem nach der Reflexionstiefe oder -güte, die sich in entsprechenden Reflexionsstufen wieder findet (Hatton \& Smith, 1995; Hänssig \& Petras, 2006).

\section{Anwendungskontext akademische Lehrpersonenbildung}

Die Lehrpersonenbildung bildet einen zentralen Einsatzbereich für ePortfolios auch und vor allem unter der Perspektive des reflexiven Lernens (Butler, 2006). Für die Analyse und Weiterentwicklung von Unterricht ist die Reflexionsfähigkeit der Lehrperson und deren Anwendung von grosser Bedeutung (Wyss, 2008). Mit Hilfe von reflexiven Methoden können Erfahrungen aus der pädagogischen Praxis analysiert und im Sinne forschenden Lernens auf der Basis wissenschaftlicher Theorien 
überprüft und verbessert werden (Wildt, 2003). Dem speziell in der ersten Phase der Lehrpersonenbildung virulenten Theorie-Praxis-Problem kann dadurch ein Stück weit entgegen gewirkt werden. Im Prozess der pädagogischen Professionalisierung kann Reflexion somit als Bindeglied zwischen pädagogischem Inhaltswissen und der Entwicklung pädagogischer Handlungskompetenzen (die sich letztlich in der Performanz der Lehrperson manifestieren) angesehen werden (Dubs, 2008). Vor diesem Hintergrund können Portfolios als Analysewerkzeug für Unterrichtspraxis eingesetzt werden und dadurch einen entscheidenden Beitrag zur Steigerung der Professionalisierung des Lehrhandelns leisten (Häcker, 2007). Auch die portfoliogestützte Explikation von Sichtweisen, Subjektiven Theorien, Überzeugungen und Werthaltungen, die für das Lehrhandeln von herausragender Bedeutung sind (Sembill \& Seifried, 2009), trägt dazu bei. Informell erworbene Kompetenzen können mit Portfolios dokumentiert und zertifiziert werden (Ness, 2009). Schliesslich dient der didaktisch fundierte Einsatz von Portfolios in der Lehrpersonenbildung auch dazu, den Ansatz und die dafür notwendigen Werkzeuge aus der akademischen Sphäre heraus in die Schulrealität zu transportieren - angehende Lehrpersonen üben dabei eine Multiplikatorenfunktion aus.

Ausgehend von diesen theoretischen Überlegungen wird im Folgenden beschrieben, wie die Implementierung von ePortfolio-Szenarios im Studium der Wirtschaftspädagogik erfolgen kann.

\section{ePortfolio-Szenarios im Studium der Wirtschaftspädagogik}

Durch den Bologna-Prozess hat sich die Wirtschaftspädagogik-Ausbildung an der Universität Bamberg nachhaltig gewandelt. Veränderte Rahmenbedingungen statt grundständigem Diplom- gibt es nun einen Masterstudiengang, der auch einen "Quereinstieg» ermöglicht - gehen mit neuen Akzentuierungen im Lehrangebot einher, in dem Veranstaltungen, die eine hohe Eigenaktivität der Studierenden erfordern, mehr Gewicht erhalten und verstärkt auf Dezentralisierung und Blended Learning gesetzt wird. Den übergreifenden Rahmen dafür bildet die Lehr-Lern-Konzeption des «Selbstorganisierten Lernens» (Sembill et al., 2007), die als konstruktivistischer Ansatz im Kern auf längerfristig angelegter gruppenorientierter Projektarbeit mit komplexen Problemstellungen basiert und im gesamten Studienprogramm Ziel, Inhalt und Methode darstellt. In diesem Kontext wurde (papierbasierte) Portfolioarbeit bereits in der Vergangenheit zur Unterstützung der sog. Schulpraktischen Übungen eingesetzt. Dabei konnten Optimierungspotenziale insbesondere hinsichtlich der Reflexion aufgezeigt werden (vgl. Brouër, 2007). Als strukturelle Voraussetzungen für eine veranstaltungsbezogene, projektorientierte Portfolioarbeit (Hornung-Prähauser et al., 2007) wurden neben einer offenen Veranstaltungsform mit hohem «Selbstorganisationsgrad» eine adäquate Betreuungsrelation (aus beiden Gründen sind Vorlesungen eher ungeeignet), Freiheits- 
grade in punkto Assessment sowie die explizite Einbindung der Portfolioarbeit in den Veranstaltungskontext identifiziert. Für den Einsatz von ePortfolios erscheint darüber hinaus ein methodischer und/oder inhaltlicher Bezug zu E-Learning notwendig. Auf dieser Basis wurden ePortfolios im Kontext zweier Veranstaltungen implementiert, die in Tab. 1 überblicksartig vorgestellt werden:

\begin{tabular}{|c|c|c|}
\hline & $\begin{array}{l}\text { Multimediale Lernumgebungen (MLU) } \\
\text { (Egloffstein, 2008) }\end{array}$ & $\begin{array}{l}\text { Hochschuldidaktisches Praktikum } \\
\text { (HDP) } \\
\text { (Egloffstein \& Oswald, 2008) }\end{array}$ \\
\hline Inhalt & $\begin{array}{l}\text { Grundlagen und Vertiefung ausgewählter } \\
\text { Themengebiete aus dem Bereich E-Learning }\end{array}$ & $\begin{array}{l}\text { Betreuung, Gestaltung, Bewertung von Klein- } \\
\text { gruppenarbeit für den Bereich } \\
\text { «Grundlagen wissenschaftlichen Arbeitens» }\end{array}$ \\
\hline $\begin{array}{l}\text { Tätigkeit der } \\
\text { Lernenden }\end{array}$ & $\begin{array}{l}\text { Bearbeitung eines eigenen Themas; Präsen- } \\
\text { tation, ePortfolio als schriftliche Seminarleis- } \\
\text { tung }\end{array}$ & $\begin{array}{l}\text { eigenverantwortliche Durchführung der Be- } \\
\text { treuung, Dokumentation über ePortfolio }\end{array}$ \\
\hline $\begin{array}{l}\text { Organisa- } \\
\text { tion } \\
\text { und Ablauf }\end{array}$ & $\begin{array}{l}\text { Präsenzseminar mit dezentralen Phasen (Ver- } \\
\text { hältnis ca. 60:40), Unterstützung durch Lern- } \\
\text { plattform }\end{array}$ & $\begin{array}{l}\text { dezentrales Praktikum; Verankerung im Rah- } \\
\text { men von Lehrveranstaltungen zur Nachberei- } \\
\text { tung der Schulpraktischen Übungen; Unter- } \\
\text { stützung durch Lernplattform }\end{array}$ \\
\hline Assessment & $\begin{array}{l}50 \% \text { Fremdbeurteilung (Portfolio) } \\
25 \% \text { Peer-Review (Präsentation) } \\
25 \% \text { Self-Assessment (Präsentation) }\end{array}$ & $\begin{array}{l}80 \% \text { Fremdbeurteilung (Portfolio) } \\
20 \% \text { Self-Assessment (Portfolio) }\end{array}$ \\
\hline $\begin{array}{l}\text { Motivation } \\
\text { für den } \\
\text { ePortfolio- } \\
\text { einsatz }\end{array}$ & $\begin{array}{l}\text { technisch und didaktisch: } \\
\text { ePortfolio als Inhalt und Methode; } \\
\text { Unterstützung kontinuierlicher themenbezo- } \\
\text { gener Arbeit v.a. in den dezentralen Phasen; } \\
\text { Aufbrechen der Linearität schriftlicher Semi- } \\
\text { narleistungen (Ersatz für Hausarbeit); Ermögli- } \\
\text { chung von Feedback und Querbezügen zwi- } \\
\text { schen den Themenbereichen; Ermöglichung } \\
\text { der zielgerichteten Einbindung zusätzlicher } \\
\text { Medientypen }\end{array}$ & $\begin{array}{l}\text { organisatorisch und didaktisch: } \\
\text { Unterstützung selbstorganisierter Tätigkeiten; } \\
\text { Dokumentation der Betreuung; Reflexion der } \\
\text { Rolle als Lernbegleiter; Portfolio als «Proxy» } \\
\text { für die Leistungsbeurteilung } \\
\text { Evaluation des Veranstaltungskonzepts }\end{array}$ \\
\hline
\end{tabular}

Tab. 1: Veranstaltungen mit ePortfolio-Einsatz im Überblick

Die unterschiedlichen Veranstaltungstypen bedingen Unterschiede in der Implementierung der ePortfolios. Die beiden resultierenden Szenarios werden in nachfolgender Tabelle (Tab. 2) gegenübergestellt. 


\begin{tabular}{|c|c|c|}
\hline & Szenario A (MLU) & Szenario B (HDP) \\
\hline $\begin{array}{l}\text { ePortfolio } \\
\text { Grund- u. Subtyp } \\
\text { (Baumgartner, } \\
\text { Himpsl, Zauchner, } \\
\text { 2009) }\end{array}$ & $\begin{array}{l}\text { Reflexionsportfolio } \\
\text { Prüfungsportfolio }\end{array}$ & $\begin{array}{l}\text { Reflexionsportfolio } \\
\text { Prüfungs- und Lernbegleitungsportfolio }\end{array}$ \\
\hline $\begin{array}{l}\text { Portfoliotyp } \\
\text { (Häcker, Liss- } \\
\text { mann, 2007) }\end{array}$ & Typ III: Showcase-Portfolio & $\begin{array}{l}\text { Typ III: Showcase-Portfolio } \\
\text { mit Elementen des Working Portfolios (Typ } \\
\text { II) }\end{array}$ \\
\hline $\begin{array}{l}\text { Portfolio- } \\
\text { bestandteile }\end{array}$ & $\begin{array}{l}\text { - Lernjournal (inkl. Einleitung und Resü- } \\
\text { mee) } \\
\text { - Projektportfolio mit Materialien } \\
\text { - Feedback durch Mitlernende }\end{array}$ & $\begin{array}{l}\text { - } \text { Einleitung } \\
\text { - } \text { Logbuch } \\
\text { - Tutoring-Berichte mit Materialien } \\
\text { - } \text { Resümee } \\
\text { - Feedback durch Mitlernende }\end{array}$ \\
\hline $\begin{array}{l}\text { Gestaltungs- } \\
\text { vorgaben }\end{array}$ & $\begin{array}{l}\text { Empfehlungen für die Portfoliostruktur } \\
\text { Orientierungsfragen für reflexive Kompo- } \\
\text { nenten }\end{array}$ & $\begin{array}{l}\text { Vorgegebene Portfoliostruktur } \\
\text { Orientierungsfragen für reflexive Kompo- } \\
\text { nenten }\end{array}$ \\
\hline $\begin{array}{l}\text { Selektions- } \\
\text { möglichkeiten }\end{array}$ & $\begin{array}{l}\text { freie Auswahl der Artefakte für das Projekt- } \\
\text { portfolio, dabei Begründung/Erläuterung } \\
\text { des Vorgehens }\end{array}$ & $\begin{array}{l}\text { Auswahl eines Tutoring-Berichts und der } \\
\text { zugehörigen Artefakte als «showcase» für } \\
\text { die Beurteilung }\end{array}$ \\
\hline $\begin{array}{l}\text { Personen- bzw. } \\
\text { Sachbezug }\end{array}$ & $\begin{array}{l}\text { sachbezogen } \\
\text { primärer Gegenstand: individuelles Pro- } \\
\text { jektthema }\end{array}$ & $\begin{array}{l}\text { sach- und personenbezogen } \\
\text { primärer Gegenstand: Betreuungs-tätigkei- } \\
\text { ten und eigene Kompetenzentwicklung }\end{array}$ \\
\hline $\begin{array}{l}\text { Lernenden- } \\
\text { perspektive }\end{array}$ & Produkt im Fokus & Produkt und Prozess im Fokus \\
\hline $\begin{array}{l}\text { Beurteilungs- } \\
\text { perspektive }\end{array}$ & summativ, Fremdbeurteilung & summativ, Fremd- und Selbstbeurteilung \\
\hline $\begin{array}{l}\text { Reflexions- } \\
\text { komponenten }\end{array}$ & $\begin{array}{l}\text { Lernjournal } \\
\text { (nicht Teil der Beurteilung) }\end{array}$ & $\begin{array}{l}\text { Einleitung, Tutoring-Berichte, Resümee } \\
\text { (Teil der Beurteilung) }\end{array}$ \\
\hline
\end{tabular}

Tab. 2: ePortfolio-Szenarios im Überblick

\section{Explorative Analyse der ePortfolio-Szenarios Forschungsfragen}

Im Rahmen einer explorativen Analyse wurden die beschriebenen ePortfolio-Szenarios im Hinblick auf die enthaltenen Reflexionen der Teilnehmenden (TN) untersucht. Vergleichbare Studien zeigen, dass der Einsatz von Portfolios nicht per se zu Reflexion führen muss, und dass deskriptive (oberflächliche) Reflexionen gegenüber kritischen (tiefgehenden) Reflexionen im Allgemeinen überwiegen (OrlandBarak, 2005). Daher werden im Folgenden sowohl Inhalt bzw. Gegenstand («Was 
wird reflektiert?») als auch Art bzw. Tiefe («Wie wird reflektiert?») der Reflexionen betrachtet. Die übergreifende Fragestellung lautet:

F1: Wie stellt sich das Gesamtbild der Reflexion bezüglich Reflexionsgegenständen und Reflexionsarten dar?

Unklar ist weiterhin, welche Rahmenbedingungen welche Reflexionsarten und -inhalte begünstigen. In einer Ex-post-facto-Betrachtung werden daher auf der Basis der wesentlichen Unterschiede in den Realisierungen der Szenarios die folgenden Fragestellungen untersucht:

F2: Hat die Einbindung der Reflexionsaufträge ins Assessment Einfluss auf die Reflexion?

F3: Hat der Grad der Strukturierung der Reflexionsaufträge Einfluss auf die Reflexion?

\section{Methodik, Stichprobe, Operationalisierung}

Zur Untersuchung dieser Forschungsfragen wurde eine strukturierende quantitative Inhaltsanalyse (vgl. Mayring, 2008, 82 ff.; Bortz \& Döring, 2006, 149 ff.) durchgeführt. Als Ausgangsmaterial wurden die «reflexionshaltigen» Artefakte der ePortfolios aus MLU (Lernjournal) und HDP (Einleitende Gedanken, Tutoring-Bericht, Resümee) aus den Durchführungen im WS 07/08 und WS 08/09 von insgesamt $N=$ 140 TN herangezogen. Tabelle 3 zeigt die Verteilung der TN auf die verschiedenen Veranstaltungsdurchführungen:

\begin{tabular}{|l|l|l|l|l|l|}
\hline & MLU 07 & HDP 07 & MLU 08 & HDP 08 & gesamt \\
\hline $\begin{array}{l}\mathrm{N} \\
(\mathrm{m} / \mathrm{w})\end{array}$ & 8 & 68 & 16 & 48 & 140 \\
& $(4 / 4)$ & $(37 / 31)$ & $(11 / 5)$ & $(21 / 27)$ & $(73 / 67)$ \\
\hline
\end{tabular}

Tab. 3: Übersicht über die Stichprobe

Auf dieser Datenbasis wurde ein deduktiv entwickeltes zweistufiges Kategorienschema angewendet, das zusätzlich durch induktiv entwickelte Kategorien ergänzt wurde. Auf der ersten Ebene erfolgte eine inhaltliche Strukturierung anhand des Reflexionsgegenstandes. Auf der zweiten Ebene erfolgte eine skalierende Strukturierung hinsichtlich der Art bzw. Tiefe der Reflexion.

Das Kategoriensystem besteht aus sechs Kategorien (Reflexionsgegenstand), die in jeweils vier identische Subkategorien (Art der Reflexion) unterteilt sind. Tab. 4 erläutert die Kategorien: 


\begin{tabular}{|l|l|}
\hline Kategorie & Erläuterung \\
\hline Lerngegenstand (LG) & $\begin{array}{l}\text { Enthält Aussagen, die sich auf Inhalte und Aufgaben im Lehr-Lern-Gesche- } \\
\text { hen beziehen. }\end{array}$ \\
\hline Lernumgebung (LU) & $\begin{array}{l}\text { Enthält Aussagen, die sich auf das Arrangement von Unterrichtsmethoden, } \\
\text { Unterrichtstechniken, Lernmaterialien und Medien (vgl. Reinmann \& Mandl, } \\
\text { 2006) beziehen. }\end{array}$ \\
\hline Soziales Umfeld (SU) & $\begin{array}{l}\text { Enthält Aussagen, die sich auf am Lehr-Lern-Geschehen beteiligte Perso- } \\
\text { nen beziehen (z. B. Mitlernende, Tutoren, Lehrpersonen). }\end{array}$ \\
\hline Orientierungsrahmen (OR) & $\begin{array}{l}\text { Enthält Aussagen, die sich auf Ziele, Einstellungen und Sichtweisen des TN } \\
\text { beziehen. }\end{array}$ \\
\hline Kompetenzaussagen (KA) & $\begin{array}{l}\text { Enthält Aussagen, die sich auf Wissen, (Problemlöse-) Fähigkeiten, Fertig- } \\
\text { keiten, objektive und subjektive Kompetenz des TN beziehen. }\end{array}$ \\
\hline Lerntätigkeit (LT) & $\begin{array}{l}\text { Enthält Aussagen, die sich auf konkrete Tätigkeiten bzw. (Lern-) Handlun- } \\
\text { gen des TN beziehen. }\end{array}$ \\
\hline
\end{tabular}

Tab. 4: Operationalisierung des Reflexionsgegenstandes

Tab. 5 illustriert die zugehörigen Subkategorien beispielhaft für den Reflexionsgegenstand «Kompetenzaussagen» (KA):

Die Subkategorien sind inklusiv, d.h., übergeordnete Kategorien enthalten bzw. erweitern die jeweils untergeordneten. Dadurch beschreiben die Subkategorien vier Reflexionsstufen mit zunehmender Reflexionstiefe (vgl. Hänssig \& Petras, 2006). Die Subkategorien deskriptiv und interpretativ beschreiben dabei oberflächliche, die Subkategorien analytisch und konstruktiv tiefgehende Arten der Reflexion.

Als Analyse- bzw. Codiereinheiten wurden zusammenhängende Sinneinheiten verwendet, deren Grösse von Satzfragmenten bis zu mehreren Sätzen reicht. Insgesamt wurden 2160 Analyseeinheiten codiert. Die Zweitcodierung von 15\% des untersuchten Materials zur Prüfung der Beobachterübereinstimmung führte zu einem Cohen's Kappa von .83, was auf eine gute Inter-Rater-Reliabilität schliessen lässt (Bortz \& Döring, 2006, 277). 


\begin{tabular}{|c|c|c|}
\hline Subkategorie & Codierregel & Textbeispiel \\
\hline Deskriptiv & $\begin{array}{l}\text { Blosses Nennen von Fak- } \\
\text { ten. }\end{array}$ & $\begin{array}{l}\text { «Während des Hochschuldidaktischen Praktikums konnte ich } \\
\text { in allen Bereichen dazulernen. Die medientechnischen Kom- } \\
\text { petenzen konnte ich durch die Arbeit an meinem ePortfolio } \\
\text { weiter verbessern.» }\end{array}$ \\
\hline Interpretativ & $\begin{array}{l}\text { Zusätzlich mind. eines der } \\
\text { folgenden Kriterien: sprach- } \\
\text { liche Verallgemeinerung, } \\
\text { Wertung, Emotionale Äus- } \\
\text { serung, Darstellung von } \\
\text { Erkenntnissen. }\end{array}$ & $\begin{array}{l}\text { "lch denke, dass ich aufgrund meiner positiven Erfahrungen } \\
\text { weiter in meiner Betreuungskompetenz gestärkt wurde und } \\
\text { nehme dies als positive Entwicklung aus dem Seminar mit.» }\end{array}$ \\
\hline Analytisch & $\begin{array}{l}\text { Zusätzlich mind. eines der } \\
\text { folgenden Kriterien: Vernet- } \\
\text { zung von Fakten, Begrün- } \\
\text { dungsmuster, begründeter } \\
\text { Zielbezug, Bezug zu Vor- } \\
\text { wissen. }\end{array}$ & $\begin{array}{l}\text { «Zu Beginn des Seminars waren meine Kenntnisse bezüglich } \\
\text { E-Learning/Multimediales Lernen und deren Relevanz für } \\
\text { Unterricht und Schule eher diffus. Aufgrund meiner Beschäfti- } \\
\text { gung mit den theoretischen Grundlagen, den ePortfolios der } \\
\text { anderen TN und der intensiven Beschäftigung mit meinem } \\
\text { Thema bin nun in der Lage, qualifiziert Beiträge zum Thema } \\
\text { E-Learning zu leisten. (...) Gehe ich also von meiner Vorher- } \\
\text { Nachher-Leistung aus, stelle ich einen überdurchschnittlichen } \\
\text { Leistungszuwachs fest.» }\end{array}$ \\
\hline Konstruktiv & $\begin{array}{l}\text { Zusätzlich Zukunftsbezug } \\
\text { und mind. eines der fol- } \\
\text { genden Kriterien: «Denken } \\
\text { über den Tellerrand hin- } \\
\text { aus», Aufzeigen von Konse- } \\
\text { quenzen. }\end{array}$ & $\begin{array}{l}\text { «Die Verbesserung von Soft-Skills stellt einen wesentlichen } \\
\text { Bestandteil sowohl für die eigene Persönlichkeit im Allgemei- } \\
\text { nen als auch speziell die Tätigkeit als Lernbegleiter dar. Ich } \\
\text { beabsichtige deshalb meine sozial-kommunikativen Kompe- } \\
\text { tenzen durch das Wirken in und Beobachten von Gruppen- } \\
\text { dynamiken zu erweitern. Die (Selbst-)Reflexion steht hier an } \\
\text { erster Stelle. Positive Gruppenprozesse zu fördern und bera- } \\
\text { tend bzw. helfend zur Seite zu stehen ist hierbei wesentlich.» }\end{array}$ \\
\hline
\end{tabular}

Tab. 5: Operationalisierung der Reflexionsarten am Beispiel Kompetenzaussagen

\section{Ergebnisse}

- Reflexionsgegenstände und Reflexionsarten im Gesamtbild

Einen Gesamtüberblick über die Ergebnisse der quantitativen Inhaltsanalyse liefern die folgenden Diagramme. Abb. 1 zeigt die Zuordnung aller Codiereinheiten zu Reflexionsgegenständen über die ePortfolios der betrachteten Kurse hinweg: Der grösste Anteil der Nennungen entfällt mit 32\% auf die Kategorie LG (691 Codierungen), der kleinste Anteil mit 6\% auf die Kategorie SU (133 Codierungen). Die TN reflektieren also zum grössten Teil die Inhalte und Aufgaben in den verschiedenen Kursen und nur zu einem geringen Teil über ihr soziales (Lern-) Umfeld. Rechnet man das Soziale Umfeld «definitionsgemäss» (vgl. Reinmann \& Mandl, 2006, 615f.) der Lernumgebung zu, so wird OR mit einem Anteil von $8 \%$ (170 Co- 
dierungen) zur Kategorie mit den wenigsten Nennungen. Die TN reflektieren also auch vergleichsweise wenig über eigene Ziele, Einstellungen und Sichtweisen.

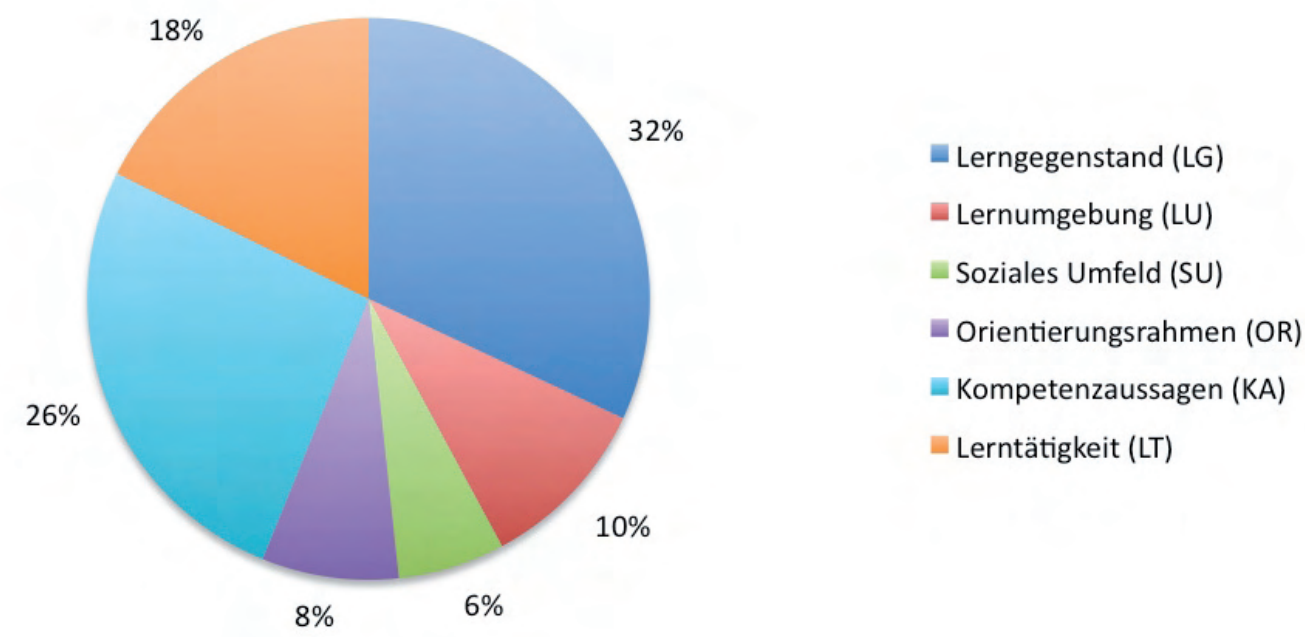

Abb. 1: Gesamtbild Reflexionsgegenstände

Die entsprechende Zuordnung aller Codiereinheiten zu den Reflexionsarten zeigt Abb. 2:

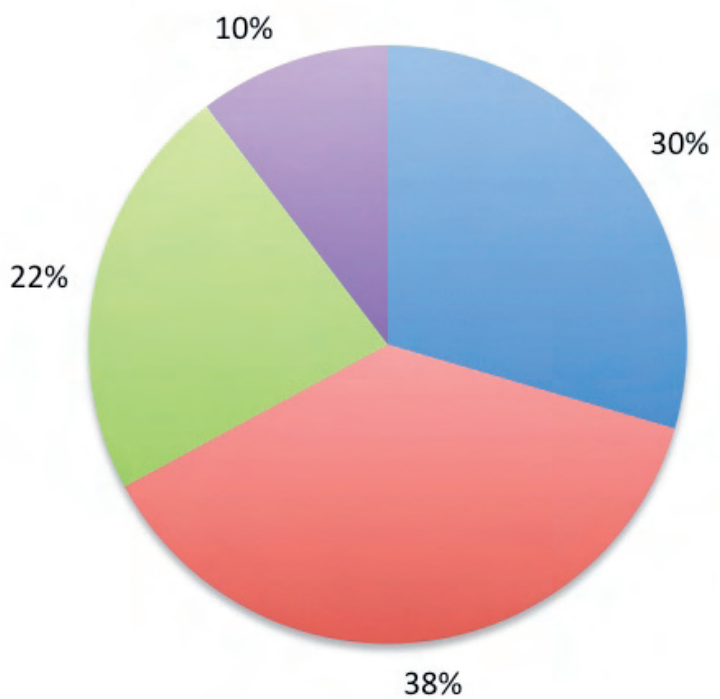

deskriptiv

interpretativ

analytisch

konstruktiv

Abb. 2: Gesamtbild Reflexionsarten 
Den grössten Anteil machen hier mit 38\% (812 Codierungen) die Nennungen der interpretativen Reflexion aus, den geringsten Anteil mit 10\% (224 Codierungen) die Kategorie der konstruktiven Reflexion. Insgesamt können mit $68 \%$ mehr als $2 / 3$ der Nennungen der oberflächlichen und nur 32\% der Nennungen der tiefgehenden Reflexion zugeordnet werden. Erwartungskonform dominieren also auch in den betrachteten Szenarios die oberflächlichen Reflexionsarten.

- Einbindung in das Assessment und Reflexion Im Rahmen dieser Fragestellung werden die unterschiedlichen Szenarios MLU (Reflexion ausserhalb des Assessments) und HDP (Reflexion als Teil des Assessments) untersucht. Für eine Gegenüberstellung kommen die Durchführungen aus dem WS 08/09 in Betracht, weil hier die Reflexionsaufträge inhaltlich und strukturell ähnlich (offene Reflexion auf Basis von Orientierungsfragen) angelegt sind. Abb. 3 zeigt eine Gegenüberstellung bzgl. der Anteile der verschiedenen Reflexionsarten.

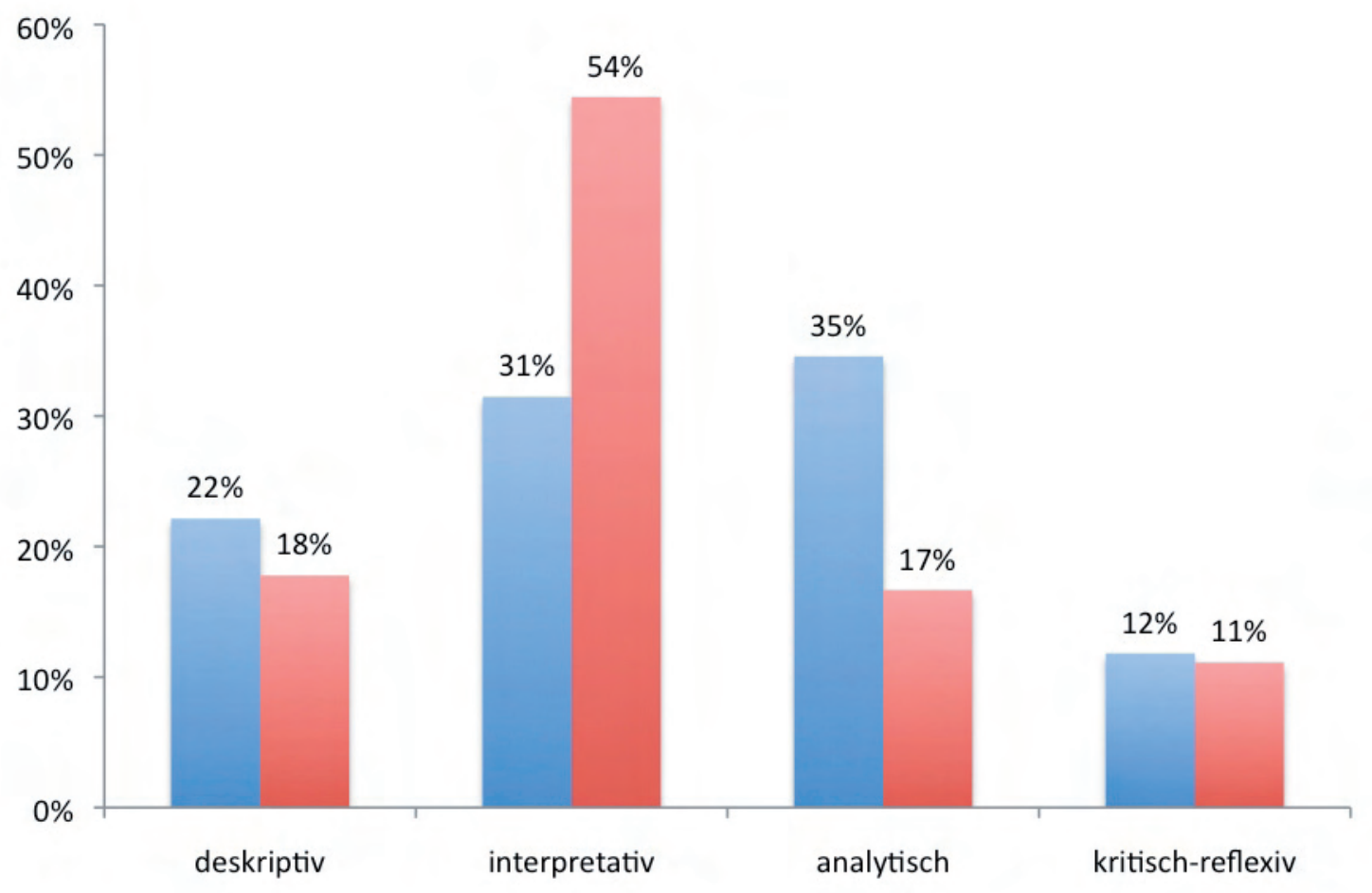

Abb. 3: Gegenüberstellung Reflexionsarten (zwischen den Szenarios) 
Wie in der Gesamtschau überwiegen auch hier für beide Szenarios die oberflächlichen Reflexionsarten (MLU: 72\%; HDP: $53 \%$ ). Augenfällig ist jedoch, dass im HDPSzenario die tiefgehenden Reflexionsarten (und hier vor allem die analytische Reflexion mit 35\%) einen weitaus höheren Stellenwert einnehmen. Darüber hinaus überwiegt im HDP-Szenario auch die mittlere Anzahl an Codierungen pro TN (MLU 08: 5.63; HDP 08: 7.81), welche als Indikator für den Umfang der Reflexionen dienen kann. Die Gegenüberstellung bzgl. der Reflexionsgegenstände zeigt Abb. 4:

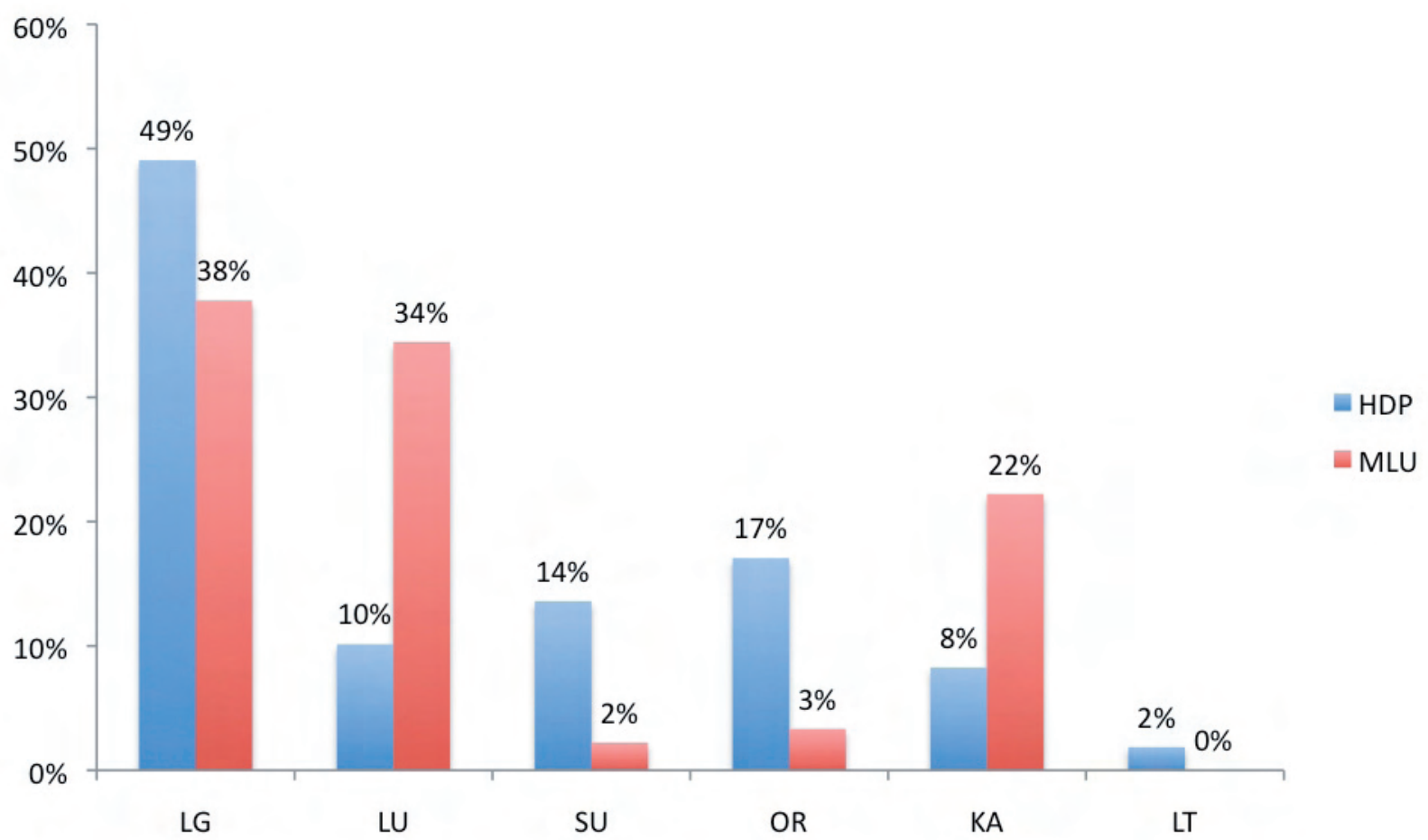

Abb. 4: Gegenüberstellung der Reflexionsgegenstände (zwischen den Szenarios)

Aufgrund der inhaltlichen Unterschiede zwischen den Veranstaltungen MLU und HDP ist für eine Gegenüberstellung der zugehörigen ePortfolio-Szenarios nur die Kategorie "Orientierungsrahmen» sinnvoll. Hier zeigt sich ein deutlich höherer Anteil im HDP (17\%).

Da die Auseinandersetzung mit Zielen, Einstellungen, Sichtweisen ein Hinweis auf tiefgehende Reflexionsprozesse sein kann, deutet dies, genau wie der höhere Anteil an tiefgehenden Reflexionsarten, auf eine höhere Reflexionsgüte im HDPSzenario hin. Ingesamt liegt somit der Schluss nahe, dass die Einbindung der Reflexion in das Assessment eine höhere Reflexionsgüte begünstigt. 
- Vorstrukturierung und Reflexion

Auf die konkrete Gestaltung von Reflexionsaufträgen bezieht sich die dritte Fragestellung. Dazu werden die HDP-Realisierungen aus dem WS 07/08 und dem WS 08/09 verglichen. Während die Reflexionen im Rahmen der PS-Berichte in HDP 07 über vorstrukturierte «Templates» mit differenzierten Einzelfragen erfolgte, wurden in HDP 08 für den äquivalenten Reflexionsauftrag übergeordnete Orientierungsfragen verwendet. Abb. 5 zeigt die Gegenüberstellung der Anteile der verschiedenen Reflexionsarten.

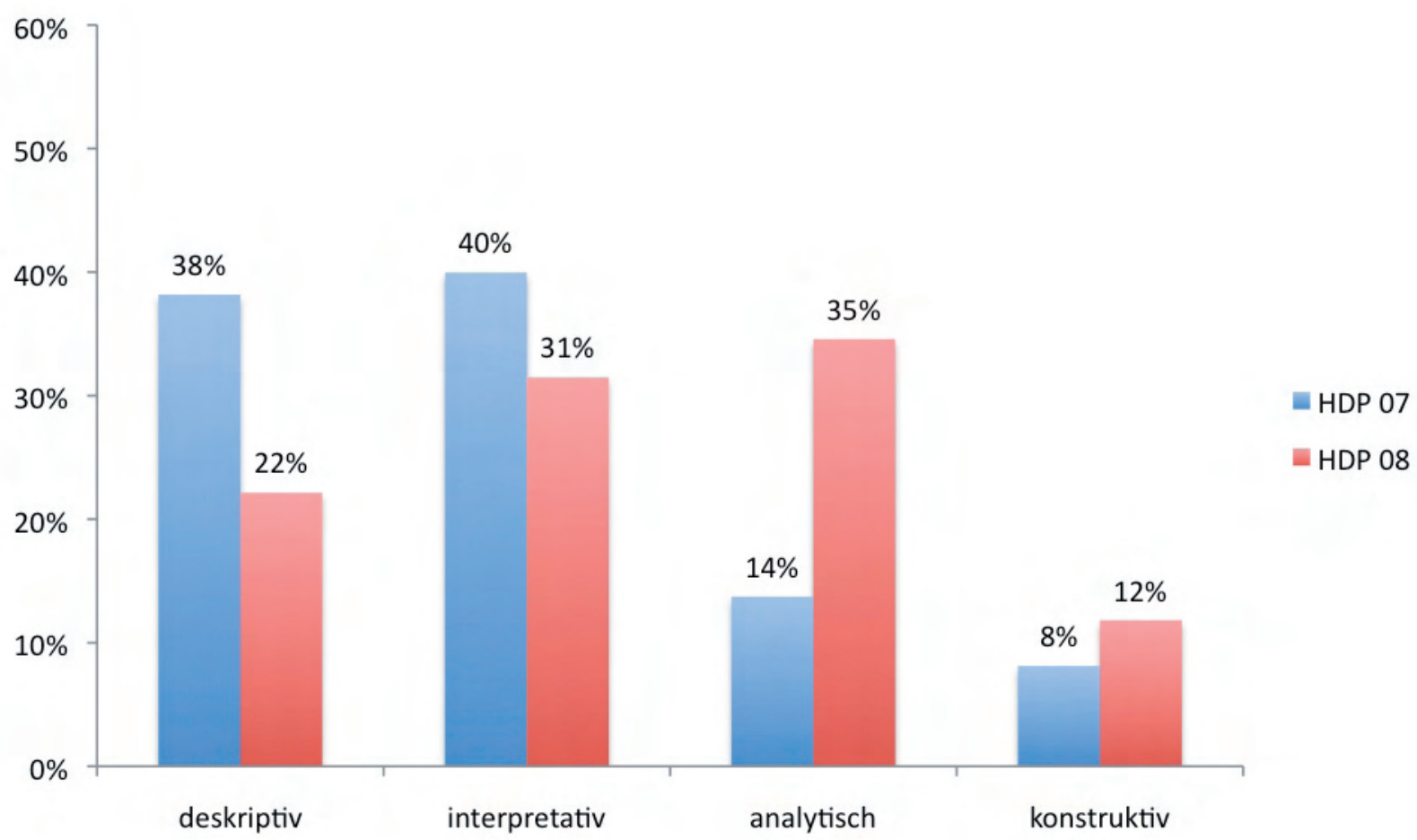

Abb. 5: Gegenüberstellung Reflexionsarten (innerhalb des HDP-Szenarios)

Deutlich fällt hier der wesentlich höhere Anteil der tiefgehenden Reflexionsarten (HDP 08: $47 \%$ vs. HDP 07: 22\%) und insbesondere der analytischen Reflexion in HDP 08 auf. Die mittlere Anzahl der Codierungen pro TN ist dagegen in HDP 07 (13.83) grösser als in HDP 08 (7.81). Die Gegenüberstellung bzgl. der Reflexionsgegenstände zeigt Abb. 6 : 


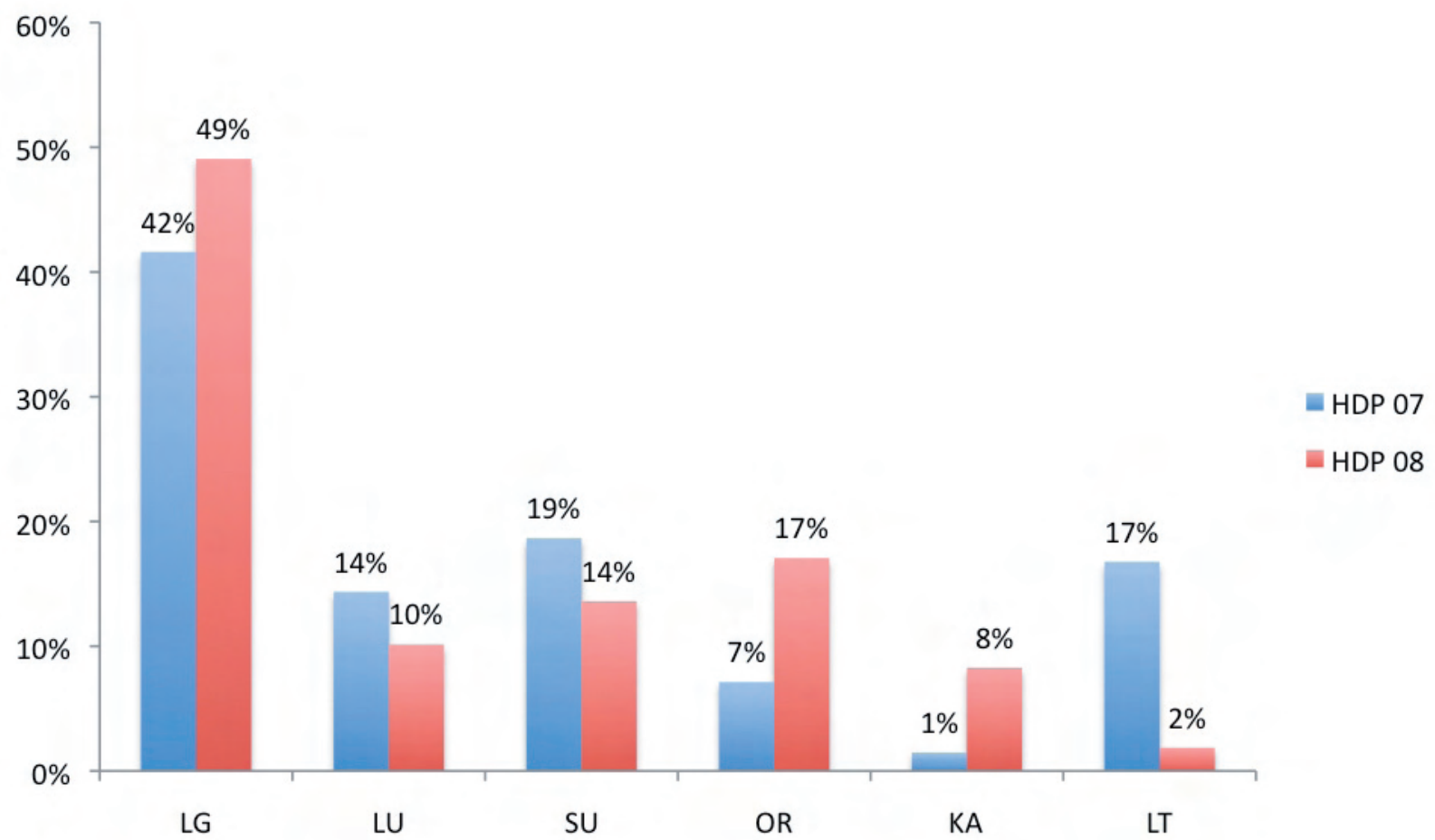

Abb. 6: Gegenüberstellung Reflexionsgegenstände (innerhalb des HDP-Szenarios)

Augenfällige Unterschiede zeigen sich insbesondere in den Kategorien «Orientierungsrahmen», für die der Anteil in HDP 08 wesentlich grösser (17\%) ist und "Lerntätigkeit», die in HDP 07 ein wesentlich stärkeres relatives Gewicht (17\%) hat.

Diese Ergebnisse lassen darauf schliessen, dass eine offene Reflexion mit Orientierungsfragen tiefer gehende Reflexionsprozesse begünstigen kann. Gleichzeitig scheint eine stärkere Vorstrukturierung dazu geeignet, den Umfang der Reflexionen (im Sinne von reflexiven vs. sonstigen Aussagen) zu erhöhen und die inhaltliche Fokussierung (HDP: Fokus auf Reflexion eigener Tätigkeiten) zu steigern. Aus methodischer Sicht bleibt jedoch anzumerken, dass diese Ergebnisse angesichts des explorativen Vorgehens und des spezifischen Kontexts nur sehr eingeschränkt interpretiert und kaum generalisiert werden können. Als Ausgangspunkt für Experimentalstudien sind die inhaltsanalytischen Ergebnisse aber durchaus von Belang.

\section{Diskussion und Ausblick}

Die vielfältigen Forschungsdesiderate im Bereich der Reflexion mit ePortfolios bedürfen weiterer Studien unter kontrollierten Bedingungen mit einer systematischen Variation der Zielvariablen. Wie die durchgeführte Analyse zeigt, könnten 
die Einbindung der Reflexion ins Assessment oder die Vorstrukturierung von Reflexionsaufträgen solche Zielvariablen sein.

Folgt man den Ergebnissen der Inhaltsanalyse dennoch etwas genauer, so erscheint es zweckmässig, Reflexionen zum Bestandteil der Leistungsbeurteilung zu machen, um tiefer gehende Reflexionsprozesse zu fördern. Dies mag widersprüchlich klingen, deckt sich aber mit der Logik des Bologna-Prozesses, nach der alle Leistungen zertifiziert werden müssen, was im Umkehrschluss nur allzu oft bedeutet: ohne Zertifizierung keine Leistung. Hier stellt sich allerdings die Frage, ob die Reflexion im Assessment nicht vielleicht nur ein (nachvollziehbares und berechtigtes) «Anpassungsverhalten» im Rahmen einer Leistungssituation darstellt. Soll die Reflexion darüber hinaus stärker fokussiert ablaufen und bestimmte inhaltliche Aspekte abdecken, so erscheint eine Vorstrukturierung der Reflexionsaufträge sinnvoll, die aber wiederum tendenziell die oberflächliche Reflexion zu begünstigen scheint. Offen bleibt bei alledem, inwieweit portfoliobasierte Reflexionen wirklich an tiefgehende Lernprozesse gekoppelt sind. Die Wendung «Papier ist geduldig» scheint hier auch für ePortfolios zu gelten - jedenfalls so lange, wie Reflexionen nicht nachweislich in neue Erkenntnisse und verändertes Handeln umgesetzt werden.

Angesichts des kurzfristigen Charakters der projektorientierten Portfolioarbeit erscheint es überdies geboten, die Portfolioarbeit über den Veranstaltungskontext hinweg auszuweiten, um so eine Reflexion zu ermöglichen, die den Professionalisierungsprozess und idealerweise auch die Persönlichkeitsentwicklung in den Blick nimmt. Dies könnte in der Wirtschaftspädagogik-Ausbildung beispielsweise durch studiengangsübergreifende Portfolioarbeit (Bachelor- und Masterphase) erfolgen, in der veranstaltungsbezogene Portfolios mit Praxisreflexionen (Kontext: Schnupperpraktikum, Schulpraktische Übungen) kombiniert und bestehende PortfolioSzenarios integriert werden. Eine Kopplung mit der mündlichen Abschlussprüfung des Master-Studiengangs würde die notwendige curriculare Integration sicherstellen. Auf diese Weise könnte das ePortfolio tatsächlich den «roten Faden» der Wirtschaftspädagogik-Ausbildung (Merkt, 2007) darstellen und zur Kompetenzentwicklung (Stratmann, Preussler \& Kerres, 2009) dienen. Insbesondere unter diesem Gesichtspunkt erscheint eine Fortführung dieses Ansatzes sinnvoll, beispielsweise in der zweiten Phase der Lehramtsausbildung (Referendariat) und darüber hinaus (Weiterbildung).

ePortfolio-Arbeit ist nicht zuletzt an die Bereitstellung einer stabilen und zukunftsfähigen technischen Infrastruktur gekoppelt. Hier bleibt abzuwarten, ob sich traditionell angelegte, zentrale ePortfolio-Systeme oder offene, Web 2.0- bzw. SocialSoftware-basierte Lösungen durchsetzen werden. Da praktisch alle momentan diskutierten ePortfolio-Werkzeuge keine elaborierten Funktionen zur Reflexionsunterstützung bieten (Hilzensauer, 2008), wird es auch in Zukunft auf die Gestaltung von Aufträgen für und Anleitungen zur Reflexion ankommen. Bis sich das ePort- 
folio auf breiter Front als digitales Reflexionswerkzeug innerhalb von «Personal Learning Environments» etablieren kann, ist - zumal im Organisationskontext der Wirtschaftspädagogik - in jedem Falle noch einiges an Anstrengung vonnöten.

\section{Literatur}

Barrett, Helen. «Electronic Portfolios.» Education and technology: An ecyclopedia. Eds. Ann Kovalchic, Kara Dawson. Santa Barbara: ABC-Clio, 2003. 271-277.

Baumgartner, Peter; Himpsl, Klaus; Zauchner, Sabine. Einsatz von E-Portfolios an (österreichischen) Hochschulen: Zusammenfassung des BMWF-Abschlussberichts «E-Portfolio an Hochschulen». Krems: Department für Interaktive Medien und Bildungstechnologien, 2009.

Boud, David; Keogh, Rosemary; Walker, David. «Promoting reflection in learning: A model.» Reflection: Turning experience into learning. Eds. David Boud, Rosemary Keogh, David Walker. London: Kogan Page, 1985. 18-40.

Bortz, Jürgen; Döring, Nicola. Forschungsmethoden und Evaluation für Humanund Sozialwissenschaftler. Heidelberg: Springer, 2006.

Brouër, Birgit. «Portfolios zur Unterstützung der Selbstreflexion - Eine Untersuchung zur Arbeit mit Portfolios in der Hochschullehre.» Lernprozesse dokumentieren, reflektieren und beurteilen. Lerntagebuch und Portfolio in Bildungsforschung und Bildungspraxis. Hrsg. v. Michaela Gläser-Zikuda u. Tina Hascher. Bad Heilbrunn: Klinkhardt, 2007. 235-265.

Butler, Philippa. A Review Of The Literature On Portfolios And Electronic Portfolios. (2006): http://eduforge.org/docman/view.php/142/1101/ePortfolio Project Research Report.pdf (22.7.2009).

Dewey, John. How we think. Boston: Heath, 1910.

Dilger, Bernadette. Der selbstreflektierende Lerner: Eine wirtschaftspädagogische Rekonstruktion zum Konstrukt der «Selbstreflexion». Paderborn: Eusl, 2007.

Dörner, Dietrich. «Selbstreflexion und Handlungsregulation: Die psychologischen Mechanismen und ihre Bedingungen.» Kausalität und Zurechnung - Über Verantwortung in komplexen kulturellen Prozessen. Hrsg. v. Weyma Lübbe. Berlin: De Gruyter, 1994. 199-222.

Doig, Bob; Illsley, Barbara; McLuckie, Joseph; Parsons, Richard. «Using ePortfolios to Enhance Reflective Learning and Development.» Handbook of Research on ePortfolios. Eds. Ali Jafari and Catherine Kaufman. Hershey: Idea Group, 2006. 158-167.

Dubs, Rolf. "Lehrerbildung zwischen Theorie und Praxis.» Pädagogische Professionalität als Gegenstand empirischer Forschung. Hrsg. v. Eva-Maria Lankes. Münster: Waxmann, 2008. 11-27.

Egloffstein, Marc. «Didaktische Variationen im Blended Learning - Lernplattformen als Konstruktionswerkzeuge.» Workshop Proceedings der Tagungen Mensch \& Computer 2008, DeLFI 2008 und Cognitive Design 2008. Hrsg. v. Ulrike Lucke, 
Martin Christof Kindsmüller, Stefan Fischer, Michael Herczeg u. Silke Seehusen. Berlin: Logos, 2008. 397-404.

Egloffstein, Marc; Oswald, Benedikt. «E-Portfolios zur Unterstützung selbstorganisierter Tutoren- und Tutorinnentätigkeiten.» Offener Bildungsraum Hochschule. Freiheiten und Notwendigkeiten. Hrsg. v. Sabine Zauchner, Peter Baumgartner, Edith Blaschitz u. Andreas Weissenbäck. Münster: Waxmann, 2008. 93-112.

Gläser-Zikuda, Michaela. «Potenziale und Grenzen von Lerntagebuch und Portfolio im Bildungsbereich.» Empirische Pädagogik 21.2 (2007): 95-100.

Hatton, Neville; Smith, David. «Reflection in teacher education: towards definition and implementation.» Teaching and Teacher Education 11.1 (1995): 33-49.

Häcker, Thomas. «Portfolio als Instrument der Kompetenzdarstellung und reflexiven Lernprozesssteuerung.» Berufs- und Wirtschaftspädagogik online - bwp@ 8 (2005): http://www.bwpat.de/ausgabe8/haecker_bwpat8.shtml (22.7.2009).

Häcker, Thomas. "Vielfalt der Portfoliobegriffe. Annäherung an ein schwer fassbares Konzept.» Das Handbuch Portfolioarbeit. Hrsg. v. Ilse Brunner, Thomas Häcker u. Felix Winter. Velber: Kallmeyer, 2006. 33-39.

Häcker, Thomas. «Professionalisierung des Lehrer/innenhandelns durch Professional Development Portfolios.» Erziehung und Unterricht 5-6 (2007): 382-391.

Häcker, Thomas; Lissmann, Urban. «Möglichkeiten und Spannungsfelder der Portfolioarbeit: Perspektiven für Forschung und Praxis.» Empirische Pädagogik 21.2 (2007): 209-239.

Hänssig, Andreas; Petras, Anneliese. «Arbeit mit Portfolio in Schulpraktischen Studien - Planung, Umsetzung und Ergebnisse.» Portfolio und Reflexives Schreiben in der Lehramtsausbildung. Hrsg. v. Margarete Imhof. Tönning: Der Andere Verlag, 2006. 29-56.

Hilzensauer, Wolf. «Theoretische Zugänge und Methoden zur Reflexion des Lernens. Ein Diskussionsbeitrag.» bildungsforschung 5.2 (2008): http://www.bildungsforschung.org/Archiv/2008-02/lernvermoegen (7.8.2009).

Hornung-Prähauser, Veronika; Geser, Guntram, Hilzensauer, Wolf; Schaffert, Sandra. "Didaktische, organisatorische und technologische Grundlagen von E-Portfolios und Analyse internationaler Beispiele und Erfahrungen mit E-Portfolio-Implementierungen an Hochschulen.» 2007: http://edumedia.salzburgresearch.at/images/stories/eportfolio_studie_srfg_fnma.pdf (5.6.2009).

Jarbonegg, Daniel. Der Portfolio-Ansatz in der Schülerbeurteilung der USA und seine Bedeutung für die Schülerbeurteilung in der neuen kaufmännsichen Grundbildung (NKG). Bamberg: Difo-Druck, 2004.

Jenert, Tobias. "Ganzheitliche Reflexion auf dem Weg zu Selbstorganisiertem Lernen.» bildungsforschung 5.2 (2008): http://www.bildungsforschung.org/Archiv/2008-02/selbstorganisation (15.6.2009).

Jonassen, David H.; Howland, Jane; Marra, Rose M.; Crismond, David P. Meaningful Learning with Technology. Boston: Allyn \& Bacon, 2007. 
Kolb, David. A. Experiential Learning. Experience as The Source of Learning and Development. Englewood Cliffs: Prentice Hall, 1984.

Korthagen, Fred. «Eine Reflexion über Reflexion.» Schulwirklichkeit und Lehrerbildung. Reflexion der Lehrertätigkeit. Hrsg. v. Fred Korthagen, Bob Koster, Bram Langerwerf u. Theo Wubbels. Hamburg: EB-Verlag, 2002. 55-73.

Mayring, Philipp. Qualitative Inhaltsanalyse - Grundlagen und Techniken. Weinheim: Beltz, 2008.

Melis, Erica; Homik, Martin. E-Portfolio Study - Germany. (2007): www.eportfolio. eu/resources/germany/publications/eportfolio-study-germany (20.7.2009).

Merkt, Marianne. "ePortfolios - der «rote Faden» zur Kompetenzentwicklung in Bachelor- und Masterstudiengängen.» Studieren neu erfinden - Hochschule neu denken. Hrsg. v. Marianne Merkt, Kerstin Mayrberger, Rolf Schulmeister, Angela Sommer u. Ivo van den Berk. Münster: Waxmann, 2007. 285-295.

Moon, Jennifer A. A Handbook of Reflective and Experiential Learning. London: Routledge, 2004.

Ness, Harry: «Portfolioarbeit zur Anerkennung informell erworbener Kompetenzen in der Lehrerbildung.» bildungsforschung 6.1 (2009). http://www.bildungsforschung.org/Archiv/2009-01/Portfolio (15.6.2009).

Orland-Barak, Lily. "Portfolios as evidence of reflective practice: what remains untold.» Educational Research 47.1 (2005): 25-44.

Paulson, Leon F.; Paulson, Pearl R.; Meyer, Carol A. «What Makes a Portfolio a Portfolio? Eight thoughtful guidelines will help educators encourage self-directed learning.» Educational Leadership 48.5 (1991): 60-63.

Reinmann, Gabi; Mandl, Heinz. "Unterrichten und Lernumgebungen gestalten.» Pädagogische Psychologie. Hrsg. v. Andreas Krapp u. Bernd Weidenmann. Weinheim: Beltz, 2006. 613-658.

Schön, Donald A. The Reflective Practitioner: How Professionals Think in Action. New York: Basic Books, 1983.

Sembill, Detlef; Seifried, Jürgen. «Konzeptionen, Funktionen und intentionale Veränderungen von Sichtweisen.» Lehrerprofessionalität: Bedingungen, Genese, Wirkungen und ihre Messung. Hrsg. v. Olga Zlatkin-Troitschanskaia, Klaus Beck, Detlef Sembill, Reinhold Nickolaus u. Regina Mulder. Weinheim: Beltz, 2009. 345-354.

Sembill, Detlef; Wuttke, Eveline; Seifried, Jürgen; Egloffstein, Marc; Rausch, Andreas. «Selbstorganisiertes Lernen in der beruflichen Bildung - Abgrenzungen, Befunde und Konsequenzen.» Berufs- und Wirtschaftspädagogik online bwp@ 13 (2007): http://www.bwpat.de/ausgabe13/sembill_etal_bwpat13.shtml (20.7.2009).

Seufert, Sabine; Brahm, Taiga. «E-Assessment und E-Portfolio zur Kompetenzentwicklung: neue Potenziale für Ne(x)t Generation Learning?» Ne(x)t Generation Learning: E-Assessment und E-Portfolio: halten sie, was sie versprechen? 
Hrsg. v. Taiga Brahm u. Sabine Seufert. St. Gallen: SCIL Arbeitsbericht 13, 2007. 2-26.

Stratmann, Jörg; Preussler, Annabell; Kerres, Michael. «Lernerfolg und Kompetenz: Didaktische Potenziale der Portfolio-Methode im Hochschulstudium.» Zeitschrift für Hochschulentwicklung 4.1 (2009): 90-103.

Wildt, Johannes. «Reflexives Lernen in der Lehrerbildung - ein Mehrebenenmodell in hochschuldidaktischer Perspektive.» Forschendes Lernen. Theorie und Praxis einer professionellen Lehrer/innenausbildung. Hrsg. v. Alexandra Obolenski u. Hilbert Meyer. Bad Heilbrunn: Klinkhardt, 2003. 71-84.

Wyss, Corinne. «Zur Reflexionsfähigkeit und -praxis der Lehrperson.» bildungsforschung 5.2 (2008): http://www.bildungsforschung.org/Archiv/2008-02/lehrperson (7.8.2009). 